\title{
High-Pressure X-ray Diffraction and Raman Spectroscopy of Ice VIII
}

\section{Citation}

Yoshimura, Yukihiro, Sarah T. Stewart, Maddury Somayazulu, Ho-kwang Mao, and Russell J. Hemley. 2006. High-pressure x-ray diffraction and Raman spectroscopy of ice VIII. Journal of Chemical Physics 124(2): 024502.

\section{Published Version}

http://dx.doi.org/10.1063/1.2140277

\section{Permanent link}

http://nrs.harvard.edu/urn-3:HUL.InstRepos:4727478

\section{Terms of Use}

This article was downloaded from Harvard University's DASH repository, and is made available under the terms and conditions applicable to Other Posted Material, as set forth at http:// nrs.harvard.edu/urn-3:HUL.InstRepos:dash.current.terms-of-use\#LAA

\section{Share Your Story}

The Harvard community has made this article openly available.

Please share how this access benefits you. Submit a story.

Accessibility 


\title{
High-pressure x-ray diffraction and Raman spectroscopy of ice VIII
}

\author{
Yukihiro Yoshimura, ${ }^{\text {a) }}$ Sarah T. Stewart, ${ }^{\text {b) }}$ Maddury Somayazulu, \\ Ho-kwang Mao, and Russell J. Hemley \\ Geophysical Laboratory, Carnegie Institution of Washington, Washington, DC 20015
}

(Received 12 July 2005; accepted 27 October 2005; published online 9 January 2006)

\begin{abstract}
In situ high-pressure/low-temperature synchrotron x-ray diffraction and optical Raman spectroscopy were used to examine the structural properties, equation of state, and vibrational dynamics of ice VIII. The X-ray measurements show that the pressure-volume relations remain smooth up to $23 \mathrm{GPa}$ at $80 \mathrm{~K}$. Although there is no evidence for structural changes to at least $14 \mathrm{GPa}$, the unit-cell axial ratio $c / a$ undergoes changes at 10-14 GPa. Raman measurements carried out at $80 \mathrm{~K}$ show that the $\nu_{T z} A_{1 g}+\nu T_{x, y} E_{g}$ lattice modes for the Raman spectra of ice VIII in the lower-frequency regions (50-800 $\mathrm{cm}^{-1}$ ) disappear at around $10 \mathrm{GPa}$, and then a new peak of $\sim 150 \mathrm{~cm}^{-1}$ appears at $14 \mathrm{GPa}$. The combined data provide evidence for a transition beginning near $10 \mathrm{GPa}$. The results are consistent with recent synchrotron far-IR measurements and theoretical calculations. The decompressed phase recovered at ambient pressure transforms to low-density amorphous ice when heated to $\sim 125$ K. (C) 2006 American Institute of Physics. [DOI: 10.1063/1.2140277]
\end{abstract}

\section{INTRODUCTION}

The high-pressure polymorphism of ice is a subject of continuing interest. At least 15 different stable and metastable forms [e.g., ice $\mathrm{I}_{h}, \mathrm{I}_{c}-\mathrm{XII}$, low-density amorphous ice (LDA), and high-density amorphouse ice (HDA)] have been identified so far, but many are still not fully characterized within their thermodynamic and kinetic stability ranges. ${ }^{1-5}$ Our understanding of the phase diagram of ice is incomplete, especially at low temperature and high pressure. Above $\sim 2 \mathrm{GPa}$, dense ices such as ices VII and VIII form, polymorphs that can be understood as interpenetration of low-pressure ice structures, and at very high-pressures ( $\sim 60 \mathrm{GPa})$ symmetric ice X phase forms. Much recent work focused on the transition to ice $X$, in which the protons of ice VII (or ice VIII) are centered between adjacent oxygens (hydrogen-bond symmetrization). On the other hand, the properties of ices VII and VIII, including the nature of its disorder and the possibility of new transformations, have been the subject of recent interest and are important for understanding the higher-pressure behavior. For example, different forms of ice VII and transitions outside its stability field have been observed. Hemley et al. ${ }^{5}$ indicated that at 4 $\mathrm{GPa}$ and $80 \mathrm{~K}$, HDA crystallizes into ice VII with further disorder, which they called ice $\mathrm{VII}^{\prime}$. Later, Klotz et al. ${ }^{6}$ reported that a phase closely related to ice VI and possibly ice $\mathrm{VII}^{\prime}$ can be recovered to $0.1 \mathrm{MPa}$ at temperatures below $95 \mathrm{~K}$.

A possible transition near 2-3 GPa in ice VIII (referred to as ice VIII') has been reported on the basis of neutron

\footnotetext{
a) Author to whom correspondence should be addressed. Present address Department of Applied Chemistry, National Defense Academy, 1-10-20 Hashirimizu, Yokosuka, Kanagawa 239-8686, Japan. FAX: (+) 8146844 5901. Electronic mail:muki@nda.ac.jp

${ }^{b)}$ Present address: Department of Earth and Planetary Science, Harvard University, 20 Oxford St., Cambridge, Massachusetts 02138.
}

diffraction, ${ }^{7}$ Raman, ${ }^{8}$ and far-infrared (FIR) measurements. ${ }^{9}$ Theoretical calculations suggest that the ice VIII transition could arise from differences in the way that the hydrogenbonded network relaxes relative to the non-hydrogen-bonded structure. ${ }^{10}$ Although it has been assumed that such recovered samples are structurally similar to the original stable phases, the results for ice VIII suggest that structural changes might occur on recovery and also show the importance of in situ measurements within its stability fields. There is, however, some confusion regarding the existence of VIII' ${ }^{7}$ Very recently, Klug et al. ${ }^{11}$ showed by synchrotron far-IR spectroscopy and linear response density-functional theory that a first-order thermodynamic transition may arise from subtle phonon instabilities associated with an isostructural movement of molecules in the ice VIII. Additional uncertainties remain concerning the nature of the transition, as well as for ice in general at low temperature and high pressure.

Here we report an in situ x-ray diffraction and Raman spectroscopy study of the behavior of ice VIII at $80 \mathrm{~K}$ up to $23 \mathrm{GPa}$ as a function of pressure. The primary experimental motivation was to examine the compression mechanism of ice VIII and to search for possible structural changes in the material. In the course of the investigations, we found an unexpected evidence for a structural transition in ice VIII at 10-14 GPa. Unusual changes in the $c / a$ ratio derived from the x-ray data occur and changes in the low-frequency Raman spectra are observed. The changes are distinct from the isostructural transition suggested to exist at lower pressures.

\section{EXPERIMENTAL METHODS}

Distilled and de-ionized water and pressure markers (5-10 $\mu \mathrm{m}$ gold chips for the X-ray and the ruby chips for the Raman) were held in a Mao-Bell-type diamond-anvil cell (DAC) using stainless-steel gaskets. The DAC was mounted in a cryostat equipped with a spring-loaded lever arm system, which enables both increasing and decreasing pressure 


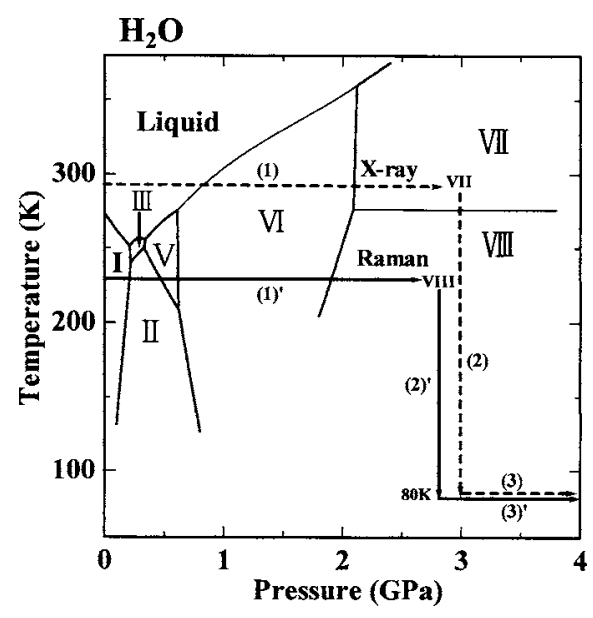

FIG. 1. Schematic experimental paths (1)-(3) and $(1)^{\prime}-(3)^{\prime}$ performed in the present study on the equilibrium phase diagram of $\mathrm{H}_{2} \mathrm{O}$. (...) Paths for the $\mathrm{x}$-ray measurements. (-) Paths for the Raman measurements.

adjustments from outside the cryostat. Two resistive heaters and a diode sensor were attached to the DAC for controlling and monitoring the temperature of the sample.

Angle dispersive x-ray-diffraction measurements using a focused synchrotron beam (HPCAT, Sector 16, Advanced Photon Source) were carried out in situ at $80 \mathrm{~K}$ with the DAC in a liquid-nitrogen cryostat. In the present experiments, the x-ray beam at $0.4218 \AA$ was collimated to $50 \mu \mathrm{m}$. The data were recorded on a MAR charge-coupled device (CCD) calibrated with a $\mathrm{CeO}_{2}$ standard. The pressure was determined from the spectral shift of the $h k l$ of the gold chip in the sample chamber of the DAC. ${ }^{12}$

Raman spectra were measured in situ up to $20 \mathrm{GPa}$ at $80 \mathrm{~K}$ with the same DAC and cryostat system used in the $\mathrm{x}$-ray measurements. Typically, the $\mathrm{OH}$-stretching vibrational mode of $\mathrm{H}_{2} \mathrm{O}$ ice can be readily followed up to $22 \mathrm{GPa}$, at which point the $\nu_{1} A_{1 g}$ and the $\nu_{3} E_{g}$ stretching modes overlap the second-order Raman band of the diamond anvils. ${ }^{13}$ The pressure was determined using the $R_{1}$ fluorescence technique. $^{14,15}$

Ice VIII was prepared by isothermally compressing water at $\sim 280 \mathrm{~K}$ over $2 \mathrm{GPa}$ so as to cross the liquid-VI-VII phase boundaries in the case of x-ray measurements or compressing ice $\mathrm{I}_{h}$ at $\sim 220 \mathrm{~K}$ over $2 \mathrm{GPa}$ in order to cross the I-II-VI-VIII phase boundaries for the Raman measurements; the cell was then cooled to liquid-nitrogen temperature of $80 \mathrm{~K}$. Ice VIII can be cooled to lower temperatures without phase change. We confirmed that the phase at $3 \mathrm{GPa}$ and $80 \mathrm{~K}$ is ice VIII by its characteristic rotational, translational, and stretching Raman spectra; ${ }^{16}$ in the case of x-ray diffraction, we confirmed by its characteristic $h k l$ indeces ( $d$ spacings). ${ }^{3,17}$ The diffraction and spectroscopic data were collected as the sample was compressed in steps to $\sim 20 \mathrm{GPa}$. We paid special attention to isothermal compression at $80 \mathrm{~K}$. The experimental paths are shown schematically in Fig. 1.

\section{RESULTS}

\section{A. X-ray diffraction}

Ice VIII has a tetragonal structure and consists of two interpenetrating cubic ice lattices with hydrogen bonds pass-

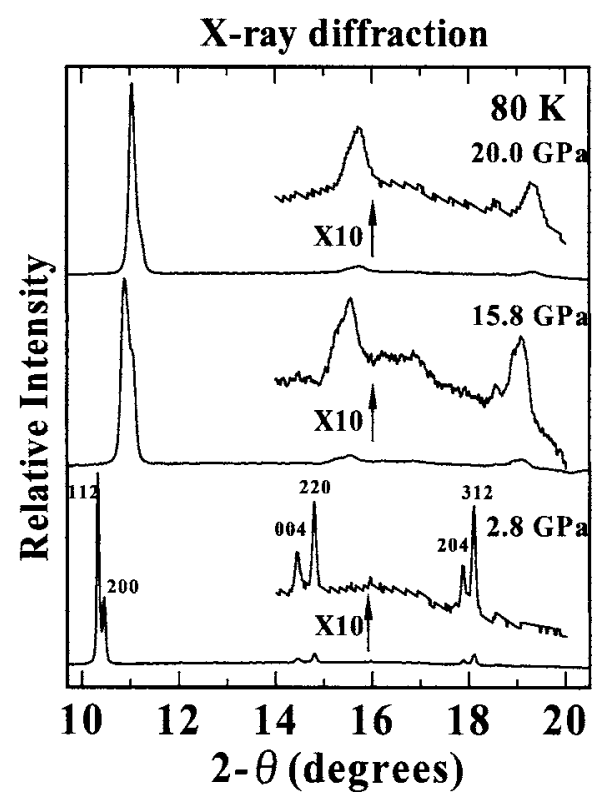

FIG. 2. In situ X-ray-diffraction patterns of ice VIII measured at different pressures at $80 \mathrm{~K}$. The high angle parts are enlarged by a factor of 10 .

ing through the center of the water hexamers and no connecting hydrogen bonds between the sublattices. The lattice parameters for the tetragonal cell are $a=4.4493 \AA$, and $c$ $=6.413 \AA$ at $2.6 \mathrm{GPa}$ and $295 \mathrm{~K},{ }^{18}$ containing eight water molecules per unit cell. We found good agreement with the reported results, ${ }^{17,19,20}$ with six-characteristic diffraction peaks below $20^{\circ}$ at $2.8 \mathrm{GPa}$ (112), (200), (004), (220), (204), and (312). To understand the high-pressure behavior of ice VIII, we measured x-ray diffraction on isothermal compression at $80 \mathrm{~K}$. Representative X-ray-diffraction patterns of ice VIII are shown in Fig. 2. Upon compression, the tetragonal doublets tend to merge into one broad peak. At 15.8 GPa, the peak splittings disappear. The patterns above $14 \mathrm{GPa}$ differ from the original ice VIII phase. It also differs from other ice phases including ice VII' ${ }^{5}$ Ice VII' is formed from HDA, ${ }^{21}$ which in turn is made from by isothermal compression of ice $\mathrm{I}_{h}$ at about $1 \mathrm{GPa}$ and $77 \mathrm{~K} .{ }^{5}$ Although the disappearance of the peak splittings can be attributed to pressure gradients (nonhydrostatic environment) at higher pressures, we feel that relatively good hydrostatic conditions were achieved from the reproducibility of the results. Also, Raman results shown below give strong support for a transition that is intrinsic to the material. The fact that the two different pressure scales (ruby for Raman and Au for x-ray) show that the changes occur over the same pressure interval.

The lattice parameters $a$ and $c$ for a tetragonal cell determined from the diffraction patterns give the pressure dependence of the axial ratio $c / a$ for the unit cell (also called the deformation parameter). The results together with the $d$ spacing are shown in Fig. 3. The $a$ and $c$ values decrease smoothly with pressure as shown [Fig. 3(b)]. The lower value of $d a / d P$ relative to $d c / d P$ may be related to the fact that at the transition from tetragonal ice VIII to cubic ice $\mathrm{X}$ at $60 \mathrm{GPa}^{22,23}$ the $c / a$ ratio reaches 2 . There is a small but distinct discontinuity in $c / a$ between 11 and $14 \mathrm{GPa}$ [Fig. $3(\mathrm{c})]$. Although the errors in the derived values in $c / a$ around 

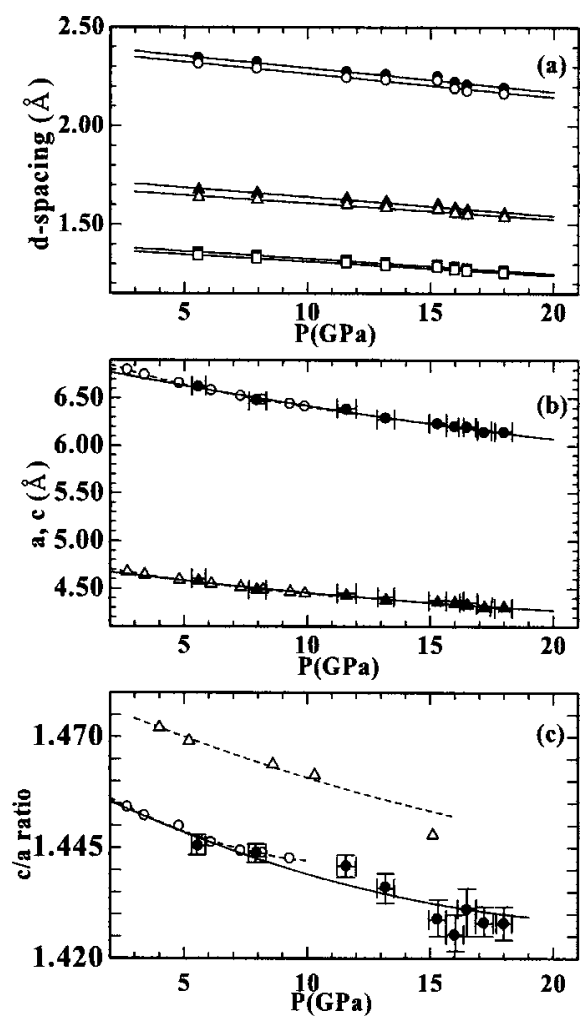

FIG. 3. (a) Pressure dependence of the $d$ spacings for ice VIII at $80 \mathrm{~K}$ ๑:(112), ○:(200), $\mathbf{\Lambda}:(004), \square:(220), \boldsymbol{\square}:(204), \square:(312)$. (b) Pressure dependence of the $a$ and $c$ for ice VIII at $80 \mathrm{~K}$. $\bullet$ and $\boldsymbol{\Delta}$ show the present results at $80 \mathrm{~K}$, and $\bigcirc$ and $\square$ represent for the experimental values by Besson et al. at $265 \mathrm{~K}$ (Ref. 18), where $\boldsymbol{\Delta}$ and $\square$ denote $a$ value, whereas $\bigcirc$ and $\bigcirc$ denote $c$ value. (c) Variation for the $c / a$ ratio with pressure. - shows the present results at $80 \mathrm{~K}$. $\bigcirc$ represents for the experimental values at $265 \mathrm{~K}$, and $\square$ is the value at $0 \mathrm{~K}$ obtained from the Hartree-Fock calculation by Besson et al. (Ref. 18) The solid and dashed lines are guides to the eyes. These are also showing that the anomaly occurs at 10-14 GPa.

the region of the discontinuity are $0.2-0.3$, the amplitude of the discontinuity is close to scatter in the data. On the other hand, there is a distinct change in the equation of state. As is mentioned above, this change in the slope of $c / a$ is well below the phase change from ice VIII to ice X. The results suggest a structural change in ice; the reslt is further explored by Raman data discussed below.

Figure 4 shows the $P-V$ equation of state (EOS) data determined from diffraction data. The results of earlier studies on ice VIII (Ref. 18) (300 K) as well as the earlier 300 and $100 \mathrm{~K}$ Birch-Murnaghan (BM) EOS fits are also shown. ${ }^{18}$ We note that the $P-V$ curve of Besson et al. was calculated from the in situ x-ray measurements of ice VII from Ref. 24, because no independent pressure calibrant was used. No sharp discontinuities are observed in the $P-V$ data from this study.

To proceed further, our $P-V$ data were fitted with a BM (Refs. 18, 25, and 26) and Vinet EOS (Ref. 27) functional forms. The fitting results with and without fixing the molar volume $V_{0}$ of $12.1 \mathrm{~cm}^{3} / \mathrm{mol}$ (Ref. 17) at ambient pressure are summarized in Table I. We found that the BM EOS deviates below $14 \mathrm{GPa}$ and the data do not fit well the data in comparison to the data of the Vinet EOS. In addition, if we do not fix the molar volume at ambient pressure $V_{0}$ to $12.1 \mathrm{~cm}^{3} / \mathrm{mol},{ }^{17}$ a smaller $K_{0}$ value is obtained. The thick

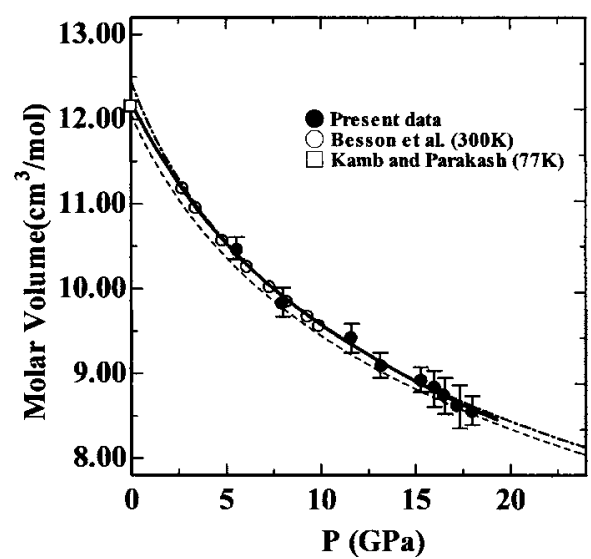

FIG. 4. Pressure dependence of the molar volume for ice VIII at $80 \mathrm{~K}$. Present data on ice VIII at $80 \mathrm{~K}$. The thick solid line is the equation of state fitted with a Vinet-type equation of state (Ref. 27), $K_{0}=27.9 \mathrm{GPa}, K_{0}^{\prime}=3.6$, and $V_{0}=12.1 \mathrm{~cm}^{3} / \mathrm{mol}$. $\bigcirc$ : The experimental values on ice VIII at $300 \mathrm{~K}$ by neutron-diffraction study by Besson et al., (Ref. 18), a dotted chain line shows the equation of state fitted with a Birch-Murnaghan (BM)-type equation of state. (Refs. 18 and 25) The dashed line shows the calculated data from the equation of state on ice VIII at $100 \mathrm{~K}$ by Besson et al. (Ref. 18) fitted with a BM equation of state. (Ref. 18) $\square$ :unpublished work by Kamb and Prakash cited in Ref. 17.

curve in Fig. 4 shows the fit with a bulk modulus $K_{0}$ $=27.9( \pm 0.3) \mathrm{GPa}$, a pressure derivative $K_{0}^{\prime}=3.6( \pm 0.5)$, and $V_{0}=12.1 \mathrm{~cm}^{3} / \mathrm{mol}$ with the Vinet EOS.

The EOS of $\mathrm{H}_{2} \mathrm{O}$ ice VIII at lower temperatures is not well established, and it is useful to compare our EOS results for ice VIII with the other phases of ice. Table II compares the best-fit parameters, zero-pressure isothermal bulk modulus $K_{0}$, its pressure derivative $K_{0}^{\prime}$, and also the zero-pressure molar volume for most ice polymorphs ${ }^{18,24,28-31}$ though the theoretical analyses of the experimental methods and data are not the same throughout.

\section{B. Raman spectroscopy}

The Raman spectra of ice VIII on isothermal compression at $80 \mathrm{~K}$ up to $20 \mathrm{GPa}$ are shown in Fig. 5 for the OH-stretching vibrational mode, and Fig. 6 for the lowfrequency rotational and translational modes. The frequencies of the vibrational bands are plotted as a function of pressure in Figs. 7 and 8. All the fundamental Raman-active vibrations were identified by Wong and Whalley ${ }^{16}$ using samples recovered at $77 \mathrm{~K}$ and $0.1 \mathrm{MPa}$. In the $\mathrm{OH}-$ stretching regions, $\mathrm{H}_{2} \mathrm{O}$ ice has three symmetrical bands identified as the $\nu_{1} A_{1 g}, \nu_{3} E_{g}$, and $\nu_{1} B_{1 g}$ fundamentals. In the low-frequency region there are four Raman-active bands:

TABLE I. Summary of the equation of state fits; molar volume $V_{0}$, isothermal bulk modulus $K_{0}(\mathrm{GPa})$, and its pressure derivative $K_{0}^{\prime}\left(d K_{0} / d P\right)$ with and without fixing the $V_{0}$ value of $12.1 \mathrm{~cm}^{3} / \mathrm{mol}$ (Ref. 19) at $0 \mathrm{GPa}$.

\begin{tabular}{cccc}
\hline \hline EOS & $\begin{array}{c}\text { Molar volume } \\
V_{0}\left(\mathrm{~cm}^{3} / \mathrm{mol}\right)\end{array}$ & $\begin{array}{c}\text { Isothermal bulk modulus } \\
K_{0}(\mathrm{GPa})\end{array}$ & $\begin{array}{c}K_{0}^{\prime} \\
d K_{0} / d P\end{array}$ \\
\hline Vinet & $12.4 \pm 0.2$ & $21.7 \pm 1.9$ & $4.7 \pm 0.6$ \\
BM & $12.2 \pm 0.1$ & $28.9 \pm 0.2$ & $3.4 \pm 0.2$ \\
Vinet & 12.1 & $27.9 \pm 0.3$ & $3.6 \pm 0.5$ \\
BM & 12.1 & $28.9 \pm 0.4$ & $3.5 \pm 0.4$ \\
\hline
\end{tabular}


TABLE II. Comparison of the parameter values, molar volume $V_{0}$, isothermal bulk modulus $K_{0}(\mathrm{GPa})$, and its pressure derivative $K_{0}^{\prime}\left(d K_{0} / d P\right)$ with some ice polymorphs.

\begin{tabular}{cccccc}
\hline \hline Phases & $\begin{array}{c}\text { Temperature } \\
(\mathrm{K})\end{array}$ & $\begin{array}{c}\text { Molar volume } \\
V_{0}\left(\mathrm{~cm}^{3} / \mathrm{mol}\right)\end{array}$ & $\begin{array}{c}\text { Isothermal bulk modulus } \\
K_{0}(\mathrm{GPa})\end{array}$ & $\begin{array}{c}K_{0}^{\prime} \\
d K_{0} / d P\end{array}$ & Reference \\
\hline $\mathrm{I}_{h}$ & 248 & 19.56 & 9.2 & 5.5 & $\mathrm{a}$ \\
II & 0 & 14.52 & 16.18 & 6.0 & $\mathrm{~b}$ \\
III & 253 & 15.91 & 7.55 & 8.1 & $\mathrm{c}$ \\
VI & 253 & 15.58 & 13.08 & 6.8 & $\mathrm{c}$ \\
VII & 300 & $12.3 \pm 0.3$ & $23.7 \pm 0.9$ & $4.15 \pm 0.7$ & $\mathrm{~d}$ \\
VIII & 100 & 12.06 & 23.6 & 4.48 & $\mathrm{e}$ \\
VIII & 87 & 12.04 & 24.3 & 4.5 & $\mathrm{f}$ \\
VIII & 80 & 12.1 & $27.9 \pm 0.3$ & $3.6 \pm 0.5$ & Present \\
& & & & & results \\
\hline
\end{tabular}

${ }^{\mathrm{a}}$ Reference 28 . The $K_{0}$ and $K_{0}^{\prime}$ values were calculated using auxiliary data on thermal expansion coefficient and specific heat.

${ }^{\mathrm{b}}$ Reference 29. The structural parameters for ice II were calculated by ab initio simulations and fitted with a Birch-Murnaghan equation of state.

${ }^{c}$ Reference 30 . Brillouin spectroscopy was used to determine the bulk modulus from the pressure dependencies of the elastic constants.

${ }^{\mathrm{d}}$ Reference 24 . The pressure-volume data from synchrotron x-ray-diffraction techniques up to $128 \mathrm{GPa}$ were fitted with a Birch-Murnaghan equation of state.

${ }^{\mathrm{e}}$ Reference 18. Isothermal equation of state (Birch-Murnaghan equation to third order) was derived from the pressure dependence of the structural parameters of deuterated ice VIII by time-of-flight neutron powder diffraction up to $10 \mathrm{GPa}$.

${ }^{\mathrm{f}}$ Reference 31 . The molar volume vs pressure of ice VIII was fitted with a Birch-Murnaghan-type equation of state using fixed values of $V_{0}=12.04 \mathrm{~cm}^{3} / \mathrm{mol}$.

$\nu_{T z} A_{1 g}+\nu_{T x, y} E_{g}, \nu_{T z} B_{1 g}+\nu_{T x, y} E_{g}, \nu_{R x, y} E_{g}$ (wagging and rocking), and $\nu_{R z} B_{2 g}$ (twisting) modes. The present data include all the expected bands, which are used in interpretation of the high pressure behavior.

The following are immediately noticeable. (1) Intensities of the $\nu_{1} B_{1 g}$ stretching and the $\nu_{T z} A_{1 g}+\nu T_{x, y} E_{g}$ lattice modes diminish gradually with increasing pressure. Finally at above 8-10 GPa the $\nu_{T z} A_{1 g}+\nu T_{x, y} E_{g}$ disappears and the $\nu_{1} B_{1 g}$ ap-

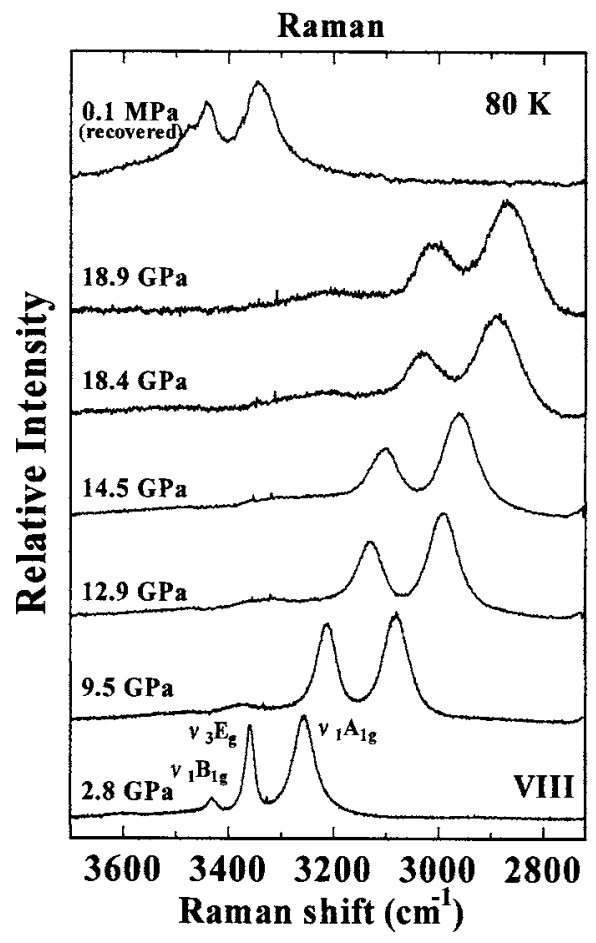

FIG. 5. In situ Raman spectral changes of ice VIII at $80 \mathrm{~K}$ in the region of the $\mathrm{OH}$-stretching vibrations as a function of pressure. pears as a very weak broadband. Raman spectral shape of the $\nu T_{z} B_{1 g}+\nu T_{x, y} E_{g}$ lattice band clearly begins to show asymmetry over this pressure. (2) Significant spectral changes were observed at around $14 \mathrm{GPa}$. A new band appears suddenly at around $150 \mathrm{~cm}^{-1}$ in the low-frequency mode (Fig. 6) and the frequency increases very rapidly with increasing pressure (Fig. 8). The relative intensity of the $\nu_{3} E_{g}$ band becomes smaller than that of the $\nu_{1} A_{1 g}$ band above this point and the half-bandwidths for both of the $\nu_{3} E_{g}$ and the $\nu_{1} A_{1 g}$ bands

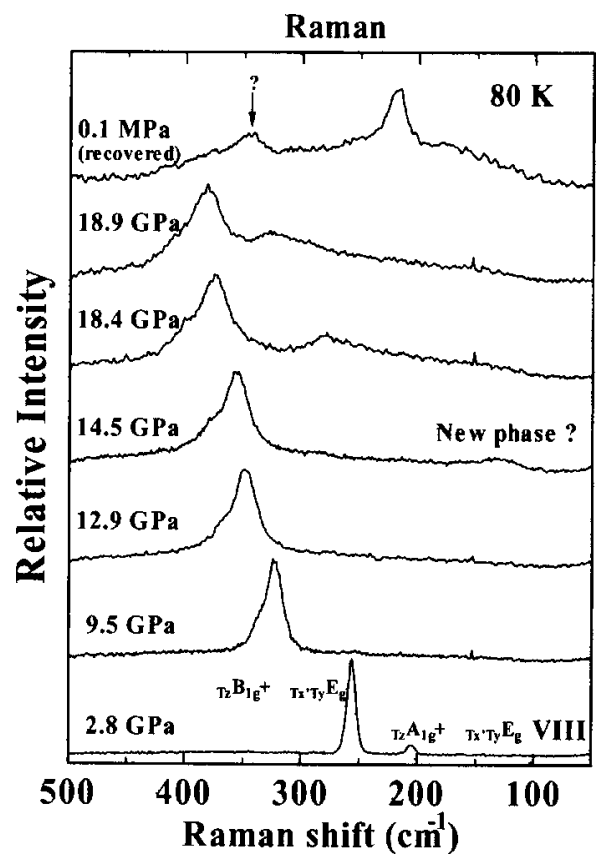

FIG. 6. In situ Raman spectral changes of ice VIII at $80 \mathrm{~K}$ in the region of the rotational and translational vibrations $\left(50-500 \mathrm{~cm}^{-1}\right)$ as a function of pressure. 


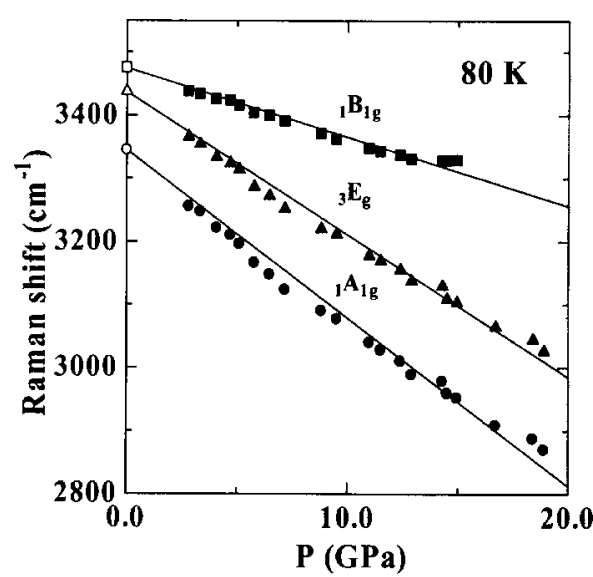

FIG. 7. Pressure dependence of the frequency of the $\mathrm{OH}$-stretching vibrational modes along path $(3)^{\prime}$. The open symbols represent the values for the retrieved state of ice VIII at $0.1 \mathrm{MPa}$. The solid lines are only guides to the eye.

become wider drastically. (3) Extrapolation of the "softmode" feature that appears above $14 \mathrm{GPa}$ down to zero frequency gives an onset pressure for the transition around 10-12 GPa (Fig. 8).

In connection with the above-mentioned results, we point out that Hirsch and Holzapfel ${ }^{13}$ reported the Raman spectra of ice VIII $\left(\mathrm{H}_{2} \mathrm{O}\right.$ and $\left.\mathrm{D}_{2} \mathrm{O}\right)$ up to $50 \mathrm{GPa}$ at $100 \mathrm{~K}$ in search of evidence for ice $\mathrm{X}$. They reported that all the bands shift very strongly and the intensities of the $\nu_{1} B_{1 g}$ stretching and the $\nu_{T z} A_{1 g}+\nu T_{x, y} E_{g}$ lattice bands decrease drastically with increasing pressure; also, above $10 \mathrm{GPa}$, the $\nu_{1} B_{1 g}$ stretching and the $\nu_{T z} A_{1 g}+\nu_{T x, y} E_{g}$ lattice bands disappear in the background. Upon further compression, the remaining bands shift nearly linear with pressure. No new peaks were reported at $100 \mathrm{~K}$ other than a new peak of the $\nu_{T 2 g}$ region above $35 \mathrm{GPa}$.

Previous Raman studies have been reported to determine the transition line between ice VII and ice VIII for $\mathrm{H}_{2} \mathrm{O}$ and $\mathrm{D}_{2} \mathrm{O}^{8,32,33}$ including the same thermodynamic path (i.e., $80 \mathrm{~K}$ isotherm and 3-27 GPa) performed in the present study. These results indicated that the transition temperature decreases linearly with pressure above $15 \mathrm{GPa}$; around $62 \mathrm{GPa}$ for $\mathrm{H}_{2} \mathrm{O}$ and $72 \mathrm{GPa}$ for $\mathrm{D}_{2} \mathrm{O}$, the transition temperature drops to $0 \mathrm{~K}$. Upon further compression, the hydrogen bonds in ices VII and VIII become symmetric at high pressures of $60 \mathrm{GPa}$, as suggested by Kamb and Davis. ${ }^{19}$ The symmetric transition was substantiated by IR spectroscopy at around 60 $\mathrm{GPa}^{22,34}$ meaning that the protons are located at the hydrogen-bond midpoints at $\sim 100 \mathrm{GPa}$ (Refs. 35-37) to $\sim 150 \mathrm{GPa}^{38}$ The appearance of a new Raman-active mode with a lower frequency at $\sim 150 \mathrm{~cm}^{-1}$ lower than the $\nu_{T z} B_{1 g}+\nu T_{x, y} E_{g}$ provides new information on the mechanism of the transition to ice $\mathrm{X}$.

As mentioned above, below 2-3 GPa, nonlinearities as a function of pressure are observed for various properties. For example, Raman frequencies measured at $3 \mathrm{GPa}$ and above do not extrapolate down to the ambient pressure values obtained from decompressed recovered samples. ${ }^{13,16}$ A phase (called VIII') having no change in space group relative to ice VIII was proposed on the basis of neutron-scattering mea-

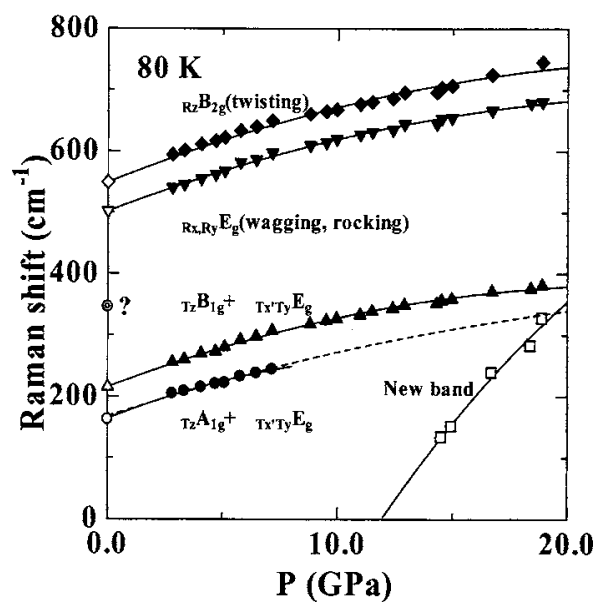

FIG. 8. Pressure dependence of the frequency of the low-frequency region along the isothermal path $(3)^{\prime}$. The open symbols represent the values for the retrieved state of ice VIII at $0.1 \mathrm{MPa}$. The double circle symbol with the question mark is the value for another new Raman peak that appears in the retrieved state (see text). The solid lines are only guides to the eye. The dashed line corresponds to the far IR data at $85 \mathrm{~K}\left(E_{u}\right.$ is the translational vibrational mode) by Klug et al. (Ref. 11).

surements when the pressure was lowered below $4 \mathrm{GPa}$ at $85 \mathrm{~K}^{7}$ The observation suggested that the transition may be second or higher order. However, subsequent first-principles calculations did not show an abrupt change in the $A_{1 g}$ internal symmetric $\mathrm{OH}$-stretching mode near the region of the structural relaxation. ${ }^{10}$ Tse and Klug ${ }^{10}$ stated that two ice $I_{c}$ lattices of ice VIII move along the $c$ axis in opposite directions and remain rigid in this process. As shown in Fig. 8, the frequency of the $\mathrm{OH}$-stretching mode versus pressure curve plot appears to go smoothly to an atmospheric pressure value through there is not sufficient experimental data to indicate whether a discontinuity has occurred in this pressure range.

Very recently Klug et al. ${ }^{11}$ have reported that the frequency dependences of both the translational and librational lattice $E_{\mathrm{u}}$ modes of IR spectra are nearly linear throughout the previously reported transition region (Fig. 8). The shift of the $\nu_{T z} A_{1 g}+\nu_{T x, y} E_{g}$ bands coincides with the $E_{u}$ mode. This coincidence may arise from a negligible vibrational coupling of an actual loss of inversion symmetry, possibly due to strain. Although there is no sign of the previously suggested features, it is interesting to point out that there may be a very slight change in slope of IR band near $15 \mathrm{GPa}$ in their results. ${ }^{11}$ The change in slope in the IR mode may be associated with the structural change documented here. Klug et $a l .{ }^{11}$ also provided the theoretical calculation of the pressure dependence for translational lattice vibrations at the Brillouin-zone center. The slope of the calculated pressure dependence of the phonon contribution to the free energy at $81 \mathrm{~K}$ shows distinct change at 5-10 GPa. It was concluded that the transition to ice VIII' was first order.

\section{DISCUSSION}

Besson et $a l .^{18}$ investigated the pressure dependence of the structural parameters of deuterated ice VIII by timeof-flight neutron diffraction up to $10 \mathrm{GPa}$. They reported the variations of $a$ and $c$ parameters of tetragonal ice VIII at $265 \mathrm{~K}$ together with the variations at $0 \mathrm{~K}$ as results of the 
Hartree-Fock calculation. We plotted all the Besson et al. data for comparison in the Figs. 3(b) and 3(c). There is good agreement in the pressure dependence of the $c / a$ between our data and their values within experimental error. It is interesting to point out that although the three data sets of 15.1, 24.2, and $36.9 \mathrm{GPa}$ at $0 \mathrm{~K}$ [not shown in Fig. 3(c)] are outside the range of their calculations and were not used for the discussion in their paper, ${ }^{18}$ their calculated values show clear changes in the $c / a$ versus pressure curve around $14 \mathrm{GPa}$.

The combined high-pressure/low-temperature Raman and $\mathrm{x}$-ray results suggest that the transformation in ice VIII starts as low as $10 \mathrm{GPa}$ and is complete around $14 \mathrm{GPa}$. Under increasing pressure at low temperatures, displacements and the dipole-dipole interactions between the two ice $\mathrm{I}_{c}$ networks occur. The $\nu_{T z} A_{1 g}$ normal mode involves the relative displacement of the two substructures along the $z$ axis. Initially, the intranetwork $\mathrm{O}-\mathrm{O}$ distances are longer to allow for the interpenetration, but upon compression over the threshold value of $\sim 14 \mathrm{GPa}$ steric repulsion between molecules develop (see also Ref. 10). We speculate that as a result of the compensation for these "stresses" in the system together with the fact that the molecular motions at low temperature of $80 \mathrm{~K}$ should be very slow, a new phase appears. But most important point is the fact that the new Raman mode indicates that the transition is not isosymmetric; it has the appearance of a zone-boundary soft mode that derives intensity from some other disorder or strain. This would imply a superlattice and it could also be a weak zone-center soft mode.

Loubeyre et al. ${ }^{39}$ found that ice VII orders in a sequence of "spatially modulated (incommensurate) phases" with no distortion from the cubic structure between 2.2 and $25 \mathrm{GPa}$ at room temperature. They concluded that the modulated phases are intrinsic properties of ice VII and tend to disappear in a strained crystal or in polycrystalline samples. At lower temperatures, ice VII transforms into the closely related structure of ice VIII. There may be similar phenomenon occurring in ice VIII due to the magnitudes of the competing dipolar interactions induced by applied pressure. Unfortunately, we could not observe the weak allowed (101) peak of ice VIII, which corresponds to the forbidden $\left(\begin{array}{lll}1 / 2 & 1 / 2 & 1 / 2\end{array}\right)$ peak of ice VII indicating a periodic modulation of the structure. It is difficult to say more about any possible relationship between the transformed phase of ice VIII at around $14 \mathrm{GPa}$ in the present observations and the modulated phase of ice VII, of which the detailed arrangement of hydrogen atoms is unclear.

As for the existence of a possible discontinuity at lower pressure $(<3 \mathrm{GPa})$, we note that no changes were observed recently by high-pressure far-IR spectroscopy. ${ }^{11}$ The existence of a transition at these low pressures has also been questioned on the basis of recent first-principles calculations. ${ }^{40}$ When we retrieve the new phase at $0.1 \mathrm{MPa}$, another new Raman peak appears at $\sim 350 \mathrm{~cm}^{-1}$ (Figs. 6 and 8). We propose that there may be a kind of "memory effect" of the new phase in the recovered sample. Klug et al. ${ }^{41}$ reported that "normal" ice VIII transforms to LDA when heated to $\sim 125 \mathrm{~K}$ at ambient pressure (temperature-induced amorphization). It has been suggested on the basis of theoretical calculations that the phonon collapse accompanying with the mechanical instability is a precursor of the hydrogen-bond network reconstruction (i.e., amorphization). ${ }^{42}$ Interestingly, the recovered phase was also observed to transform to LDA above $120 \mathrm{~K}$ when heated at $0.1 \mathrm{MPa}$. We also know that stress has large effects due to the high strength of ice. ${ }^{18,31}$ The identification of transition in ice VII at these pressures also needs to be examined for future work, for example, using radial diffraction techniques. ${ }^{43}$

\section{CONCLUSIONS}

The present study has provided new information on the stability limits of ice VIII. The phase has regions of thermodynamic stability (e.g., $<278 \mathrm{~K}$ and $>2.2 \mathrm{GPa}$ ) and can be metastable at $0.1 \mathrm{MPa}$ at $<135 \mathrm{~K}$; it is also completely unstable at $0.1 \mathrm{MPa}$ and $300 \mathrm{~K}$. Under pressure and low temperature $(80 \mathrm{~K})$, we document structural changes between 10 and $14 \mathrm{GPa}$ by x-ray diffraction and Raman spectroscopy. On compression at these temperatures, the $\nu_{T z} A_{1 g}+\nu T_{x, y} E_{g}$ lattice mode observed in the Raman spectrum at $50-800 \mathrm{~cm}^{-1}$ disappears at around $10 \mathrm{GPa}$ and a new peak of $\sim 150 \mathrm{~cm}^{-1}$ appears that implies structural transformation that is complete by $14 \mathrm{GPa}$. Concordant with the Raman results, the pressure dependence of the $c / a$ axial ratio is anomalous at 10-14 GPa. The phase can be quenched to 0.1 $\mathrm{MPa}$ at $80 \mathrm{~K}$, and transforms to LDA, and then to ice $\mathrm{I}_{h}$ when heated at $0.1 \mathrm{MPa}$, like the ordinary ice VIII. In addition to adding to our current knowledge of ice VIII, the present study provides useful baseline Raman and x-raydiffraction data for dense ices. The detailed natures of these ices are longstanding problems in ice physics, and have implications for the outer solar system. The results should also stimulate new theoretical studies of the interesting behavior of the $\mathrm{H}_{2} \mathrm{O}$ system as functions of pressure and temperature.

\section{ACKNOWLEDGMENTS}

We thank D. Häusermann, Y. Meng, Y. Ding, E. Rod, M. $\mathrm{Hu}$, and V. O'Connor for technical assistance, and M. Phillips for help with the manuscript. This work was supported by NASA (NAI), NSF-EAR, and NSF-DMR. The HPCAT facility supported by DOE-BES, DOE-NNSA, NSF, DODTACOM, and by the W.M. Keck Foundation. HPCAT is a collaboration among the Carnegie Institution, Lawrence Livermore National Laboratory, the University of Hawaii, the University of Nevada Las Vegas, and the Carnegie/DOE Alliance Center (CDAC).

\footnotetext{
${ }^{1}$ E. Whalley, in The Hydrogen Bond, edited by P. Schuster, G. Zundel, and C. Sandorfy (North-Holland, Amsterdam, 1976).

${ }^{2}$ P. W. Bridgman, J. Chem. Phys. 5, 964 (1937).

${ }^{3}$ B. Kamb, Trans. Am. Crystallogr. Assoc. 5, 61 (1969).

${ }^{4}$ P. V. Hobbs, Ice Physics (Clarendon, Oxford, 1974).

${ }^{5}$ R. J. Hemley, L. C. Chen, and H. K. Mao, Nature (London) 338, 638 (1989).

${ }^{6}$ S. Klotz, J. M. Besson, G. Hamel, R. J. Nelmes, R. J. Loveday, and W. G. Marshall, Nature (London) 398, 681 (1999).

${ }^{7}$ J. M. Besson, S. Klotz, G. Hamel, W. G. Marshall, R. J. Nelmes, and J. S. Loveday, Phys. Rev. Lett. 78, 3141 (1997).

${ }^{8}$ J. M. Besson, M. Kobayashi, T. Nakai, S. Endo, and P. Pruzan, Phys. Rev. B 55, 11191 (1997).
} 
${ }^{9}$ M. Kobayashi, T. Nanba, M. Kamada, and S. Endo, J. Phys.: Condens. Matter 10, 11551 (1998).

${ }^{10}$ J. S. Tse and D. D. Klug, Phys. Rev. Lett. 81, 2466 (1998).

${ }^{11}$ D. D. Klug, J. S. Tse, Z. Liu, X. Gonze, and R. J. Hemley, Phys. Rev. B 70, 1 (2004).

${ }^{12}$ S.-H. Shim, T. S. Duffy, and T. Kenichi, Earth Planet. Sci. Lett. 203, 729 (2003).

${ }^{13}$ K. R. Hirsch and W. B. Holzapfel, J. Chem. Phys. 84, 2771 (1986).

${ }^{14}$ J. C. Chervin, B. Canny, M. Guthier, and Ph. Pruzan, Rev. Sci. Instrum. 64, 203 (1993).

${ }^{15}$ H. K. Mao, P. M. Bell, J. W. Shaner, and D. J. Steinberg, J. Appl. Phys. 49, 3276 (1978); H. K. Mao, J. Xu, and P. M. Bell, J. Geophys. Res. 91, 4673 (1986)

${ }^{16}$ P. T. T. Wong and E. Whalley, J. Chem. Phys. 64, 2359 (1976).

${ }^{17}$ W. F. Kuhs, J. L. Finney, C. Vettier, and D. V. Bliss, J. Chem. Phys. 81, 3612 (1984).

${ }^{18}$ J. M. Besson, Ph. Pruzan, S. Klotz, G. Hamel, B. Silvi, R. J. Nelmes, J. S. Loveday, R. M. Wilson, and S. Hull, Phys. Rev. B 49, 12540 (1994)

${ }^{19}$ B. Kamb and B. L. Davis, Proc. Natl. Acad. Sci. U.S.A. 52, 1433 (1964).

${ }^{20}$ J. L. Finney, Acta Crystallogr., Sect. B: Struct. Sci. B51, 447 (1995).

${ }^{21}$ O. Mishima, L. D. Calvert, and E. Whalley, Nature (London) 310, 393 (1984).

${ }^{22}$ M. Song, H. Yamawaki, H. Fujihisa, M. Sakashita, and K. Aoki, Phys. Rev. B 60, 12644 (1999).

${ }^{23}$ A. F. Goncharov, V. V. Struzhkin, H. K. Mao, and R. J. Hemley, Phys. Rev. Lett. 83, 1998 (1999).

${ }^{24}$ R. J. Hemley, A. P. Jephcoat, H. K. Mao, C. S. Zha, L. W. Finger, and D. E. Cox, Nature (London) 330, 737 (1987).

${ }^{25}$ E. Wolanin, Ph. Pruzan, J. C. Chervin, B. Canny, and M. Gauthier, Phys. Rev. B 56, 5781 (1997).

${ }^{26}$ R. Jeanloz, Geophys. Res. Lett. 8, 1219 (1981).
${ }^{27}$ P. Vinet, J. Ferrante, J. R. Smith, and J. H. Rose, J. Phys. C 19, L467 (1986).

${ }^{28}$ G. W. Shaw, J. Chem. Phys. 84, 5862 (1986).

${ }^{29}$ A. D. Fortes, I. G. Wood, J. P. Brodholt, and L. Vocadlo, J. Chem. Phys. 119, 4567 (2003)

${ }^{30}$ C. A. Tulk, R. E. Gagnon, H. Kiefte, and M. J. Clouter, J. Chem. Phys. 107, 10684 (1997).

${ }^{31}$ H. Yamawaki, H. Fujihisa, M. Sakashita, A. Nakayama, and K. Aoki, Physica B 344, 260 (2004)

${ }^{32}$ Ph. Pruzan, J. C. Chervin, and B. Canny, J. Chem. Phys. 99, 9842 (1993).

${ }^{33} \mathrm{Ph}$. Pruzan, J. C. Chervin, and B. Canny, J. Chem. Phys. 97, 718 (1992).

${ }^{34}$ A. F. Goncharov, V. V. Struzhkin, M. S. Somayazulu, R. J. Hemley, and H. K. Mao, Science 273, 218 (1996).

${ }^{35}$ K. Aoki, H. Yamawaki, M. Sakashita, and H. Fujihisa, Phys. Rev. B 54, 15673 (1996).

${ }^{36}$ M. Benoit, D. Marx, and M. Parrinello, Nature (London) 392, 258 (1998).

${ }^{37} \mathrm{Ph}$. Pruzan, E. Wolanin, M. Gauthier, J. C. Chrvin, B. Canny, D. Häusermann, and M. Hanfland, J. Phys. Chem. B 101, 6230 (1997).

${ }^{38}$ A. F. Goncharov, V. V. Struzhkin, M. S. Somayazulu, R. J. Hemley, and H. K. Mao, Science 273, 218 (1996).

${ }^{39}$ P. Loubeyre, R. Letoullec, E. Wolanin, M. Hanfland, and D. Hausermann, Nature (London) 397, 503 (1999).

${ }^{40}$ K. Umemoto and R. M. Wentzcovich, Phys. Rev. B 71, 012102 (2005).

${ }^{41}$ D. D. Klug, Y. P. Handa, J. S. Tse, and E. Whalley, J. Chem. Phys. 90, 2390 (1989).

${ }^{42}$ K. Umemoto and R. M. Wentzcovitch, Phys. Rev. B 69, 180103 (2004).

${ }^{43}$ Y. Yoshimura, S. T. Stewart, M. Somayazulu, H. K. Mao, and R. J. Hemley (unpublished). 